\title{
Comunicação na transição do paciente oncológico para os cuidados paliativos: uma revisão integrativa
}

\author{
Communication in the transition from cancer patient to palliative care: an integrative review \\ Comunicación en la transición del paciente oncológico a los cuidados paliativos: una revisión \\ integradora
}

Recebido: 26/03/2021 | Revisado: 02/04/2021 | Aceito: 05/04/2021 | Publicado: 15/04/2021

Jeniffer Lopes Rodrigues da Silva

ORCID: https://orcid.org/0000-0003-4117-6697

Universidade Federal do Estado do Rio de Janeiro, Brasil E-mail: jenny.junior13@gmail.com

Sônia Regina de Souza

ORCID: https://orcid.org/0000-0001-7981-0038 Universidade Federal do Estado do Rio de Janeiro, Brasil E-mail: soniasilvio0@gmail.com

Laisa Figueiredo Ferreira Lós de Alcântara

ORCID: https://orcid.org/0000-0002-2435-7616 Universidade Federal do Estado do Rio de Janeiro, Brasil E-mail: dra.laisa@gmail.com

Eliza Cristina Macedo

ORCID: https://orcid.org/0000-0001-8824-9107 Universidade Federal do Estado do Rio de Janeiro, Brasil E-mail:macedo.unirio@gmail.com

Dasymar Martins da Silva Lucas ORCID: https://orcid.org/0000-0002-6380-6031 Universidade Federal do Estado do Rio de Janeiro, Brasil E-mail:dasy_lucas@hotmail.com Isabella Ribeiro Cardozo

ORCID: https://orcid.org/0000-0003-0481-7088 Universidade do Estado do Rio de Janeiro, Brasil E-mail: belaenfermagem@gmail.com

Maria Célia Teixeira Barbosa

ORCID: https://orcid.org/0000-0002-7490-5537 Universidade Federal do Estado do Rio de Janeiro, Brasil E-mail:celiatbarbosa@gmail.com

Lívia de Souza Câmara

ORCID: https://orcid.org/0000-0001-6848-3852 Universidade Federal do Estado do Rio de Janeiro, Brasil E-mail:liviacamara88@gmail.com

\begin{abstract}
Resumo
O objetivo foi investigar as evidências acerca da comunicação na transição do paciente oncológico para os cuidados paliativos. Realizou-se uma revisão integrativa através da estratégia PICo, cuja questão norteadora foi "Quais as evidências acerca da comunicação na transição do paciente oncológico para os cuidados paliativos? As bases de dados foram MEDLINE, LILACS e BDENF através da Biblioteca Virtual em Saúde; Pubmed, Scopus e Web of Science. Foram identificados 14 artigos publicados no período de 2014 - 2018. Através da análise temática classificou-se duas categorias temáticas: 1- Barreiras da comunicação na transição para os cuidados paliativos 2- Estratégias para uma comunicação eficaz na transição para os cuidados paliativos. As categorias temáticas abordadas evidenciaram que a comunicação na transição para os cuidados paliativos ainda é um momento difícil e complexo para os profissionais de saúde, favorecendo barreiras comunicacionais entre a tríade equipe-paciente-família. Entretanto é um momento estratégico para planejar e redesenhar objetivos de tratamento, onde estratégias como uma comunicação honesta, o mais breve possível e contínua são importantes para uma comunicação eficaz.
\end{abstract}

Palavras-chave: Oncologia; Comunicação; Cuidados paliativos.

\begin{abstract}
The objective was to investigate the evidence about the communication in the transition from cancer patient to palliative care. An integrative review was carried out through the PICo strategy, whose guiding question was "What are the evidences about communication in the transition from the cancer patient to the palliative care? The databases were MEDLINE, LILACS and BDENF through the Virtual Health Library; Pubmed, Scopus and Web of Science. A
\end{abstract}


total of 14 articles were published in the period 2014-2018. The thematic analysis classified two thematic categories: 1- Communication barriers in the transition to palliative care. 2- Strategies for effective communication in the transition to palliative care. The thematic categories discussed showed that communication in the transition to palliative care is still a difficult and complex time for health professionals, favoring communication barriers between the team-patient-family triad. However it is a strategic time to plan and redesign treatment goals, where strategies such as honest, brief and continuous communication are important for effective communication.

Keywords: Medical oncology; Communication; Palliative care.

\section{Resumen}

El objetivo fue investigar la evidencia sobre la comunicación en la transición del paciente oncológico a los cuidados paliativos. Se realizó una revisión integradora a través de la estrategia PICo, cuya pregunta orientadora fue “¿Cuál es la evidencia sobre la comunicación en la transición del paciente oncológico a los cuidados paliativos? Las bases de datos fueron MEDLINE, LILACS y BDENF a través de la Biblioteca Virtual en Salud; Pubmed, Scopus y Web of Science. Se identificaron 14 artículos publicados en el período 2014 - 2018. A través del análisis temático se clasificaron dos categorías temáticas: 1- Barreras de comunicación en la transición a cuidados paliativos 2- Estrategias para la comunicación efectiva en la transición a cuidados paliativos. Las categorías temáticas abordadas mostraron que la comunicación en la transición a los cuidados paliativos sigue siendo un momento difícil y complejo para los profesionales de la salud, favoreciendo las barreras de comunicación entre la tríada equipo-paciente-familia. Sin embargo, es un momento estratégico para planificar y rediseñar los objetivos del tratamiento, donde estrategias como la comunicación honesta, lo antes posible y continua son importantes para una comunicación eficaz.

Palabras clave: Oncología médica; Comunicación; Cuidados paliativos.

\section{Introdução}

Atualmente, o câncer tem sido um dos problemas de saúde pública mais complexos, o qual tem ganhado destaque na atenção à saúde devido a grande letalidade em países desenvolvidos e em desenvolvimento (Sawada et al., 2017). Para o biênio 2018 - 2019 são estimados 600 mil novos casos de câncer, para cada ano, onde excetuando-se o câncer de pele não melanoma, ocorrerão 420 mil novos casos (INCA, 2017).

Com o envelhecimento da população, somado aos avanços tecnológicos e científicos, que resultam em diagnósticos e tratamentos cada vez mais precoces, o número de sobreviventes com câncer tem aumentado no mundo inteiro (Desantis et al., 2014) todavia a cura nem sempre é possível, o que reflete a importância de terapêuticas a favor da melhor qualidade vida e controle de sintomas.

Nesse contexto, surgem os cuidados paliativos, os quais são designadas as pessoas que sofrem com doenças graves, avançadas e progressivas com objetivo de proporcionar alívio da dor e controle de sinais e sintomas, promovendo melhor qualidade de vida e bem-estar (Abreu, 2017). Dessa forma, os cuidados paliativos são cuidados que não visam à cura, os mesmos devem ser aplicados independente do prognóstico e em conjunto com outras terapêuticas com vista a melhorar a qualidade de vida e minimizar o sofrimento durante todo o processo (Lima \& Oliveira, 2015).

Dentre as diversas estratégias de cuidar nos cuidados paliativos, destaca-se a comunicação (Andrade, Costa \& Lopes, 2013). Essa é um pilar de grande importância, considerada uma ferramenta indispensável para proporcionar cuidado individualizado e com qualidade para que a ansiedade e conflitos possam ser solucionados, posto que o paciente em cuidados paliativos deseja ser compreendido como um ser humano além da dor física, já que possui conflitos existenciais e necessidades que fármacos e aparelhos de alta tecnologia não podem suprir (Almeida \& Garcia, 2015; Pacheco et al, 2020).

A comunicação de notícias difíceis pode ser considerada como qualquer informação que englobe mudança drástica e negativa na perspectiva de futuro de uma pessoa. Um dos tipos de comunicação de notícias difíceis é o esgotamento dos recursos de cura e preparação para cuidados paliativos. Apesar das notícias difíceis estarem pulverizadas a todo o momento ao longo do tratamento, a revelação de diagnósticos ameaçadores de vida e agravamento da doença, ainda é uma situação indesejável para muitos profissionais de saúde, por exigir um preparo psíquico e emocional para lidar com suas próprias limitações e com as angústias do paciente, impedindo os sonhos e a esperança de um futuro diferente para esse (Kumata \& Dupas, 2016; Afonso \& Minayo, 2017). 
Diante da problemática da comunicação na transição para os cuidados paliativos, o aumento cada vez maior da demanda dessa população nos serviços de saúde e a relevância da temática para a assistência, o objetivo do estudo é investigar as evidências acerca da comunicação na transição do paciente oncológico para os cuidados paliativos.

\section{Metodologia}

Realizou-se uma revisão integrativa da literatura, técnica que permite a inclusão de estudos experimentais e nãoexperimentais para uma compreensão completa do fenômeno analisado, possibilitando chegar a conclusões sobre determinado tema, a partir da síntese de estudos publicados mediante a aplicação de método sistemático que contribui para o aprofundamento do conhecimento do tema investigado (Souza, Silva \& Carvalho, 2010).

Utilizando-se da estratégia PICo (Butler et al., 2016), sendo "P" população, "I" fenômeno de interesse, "Co" contexto foi elaborada a seguinte questão norteadora para esta revisão: Quais as evidências acerca da comunicação na transição(I) do paciente oncológico(P) para os cuidados paliativos(Co)?

A busca ocorreu no período de Outubro - Dezembro de 2019. As bases de dados utilizadas foram Medical Literature Analysis and Retrieval System Online (Medline), Literatura Latino-americana e do Caribe em Ciências da Saúde (LILACS) e Base de dados de enfermagem (BDENF), através da Biblioteca Virtual em Saúde (BVS); Public Medline (Pubmed), Scopus e Web of Science (WOS). Foram utilizados os descritores dos tesauros DeCS/MeSH, conforme o Quadro 1, nas versões inglês e português a depender da base de dados utilizada, em diferentes combinações, para a verificação do título, resumo e/ou assunto.

Quadro 1 - Estratégias de busca de acordo com cada base de dados, Rio de Janeiro/RJ, 2020.

\begin{tabular}{|l|l|l|}
\hline \multicolumn{2}{|c|}{ DeCS } & \multicolumn{1}{c|}{ MeStratégia de busca } \\
\hline P & $\begin{array}{l}\text { (Paciente terminal) OR } \\
\text { (Cancerologia) OR (Oncologia } \\
\text { clínica) }\end{array}$ & (medical oncology) OR (Oncology, Clinical) OR (Oncology, Medical) OR (Clinical Oncology) \\
\hline I & $\begin{array}{l}\text { (Comunicação em saúde) OR } \\
\text { (Canal interpessoal) }\end{array}$ & $\begin{array}{l}\text { (communication) OR (Communication Programs) OR (Communications Personnel) OR } \\
\text { (misinformation ) OR (Personal Communication) OR (Communication Program) OR } \\
\text { (Communication, Personal) OR (Personnel, Communications) OR (Program, } \\
\text { Communication) OR (Programs, Communication) }\end{array}$ \\
\hline \multicolumn{2}{|c|}{ Continuação Quadro 1 - Estratégias de busca de acordo com cada base de dados, Rio de Janeiro/RJ, 2020. } \\
\hline Co & $\begin{array}{l}\text { (cuidados paliativos ) OR } \\
\text { (assistência paliativa) OR } \\
\text { (cuidado paliativo) OR } \\
\text { (tratamento paliativo) }\end{array}$ & $\begin{array}{l}\text { (Palliative care) OR (Palliative Therapy) OR (Therapy, Palliative) OR (Care, Palliative) OR } \\
\text { (Palliative Treatments) OR (Treatment, Palliative) OR (Treatments, Palliative) }\end{array}$ \\
\hline
\end{tabular}

Fonte: Autores (2020).

Os critérios de inclusão considerados foram: artigos em português, inglês e espanhol, publicados entre 2014-2018 e disponíveis na íntegra. Foram excluídos artigos duplicados; os que abordavam a comunicação com pacientes pediátricos ou somente com familiares e cuidadores - pela especificidade desse grupo populacional e pelo tratamento diferenciado na 
comunicação com a família; foram excluídos, ainda, os estudos relacionados a pacientes não oncológicos e os que não descreveram a metodologia de forma clara, além de teses e dissertações.

\section{Resultados}

A amostra final foi de 14 artigos como está detalhado na Figura 1

Figura 1 - Fluxograma de seleção de artigos elaborado com base no PRISMA.

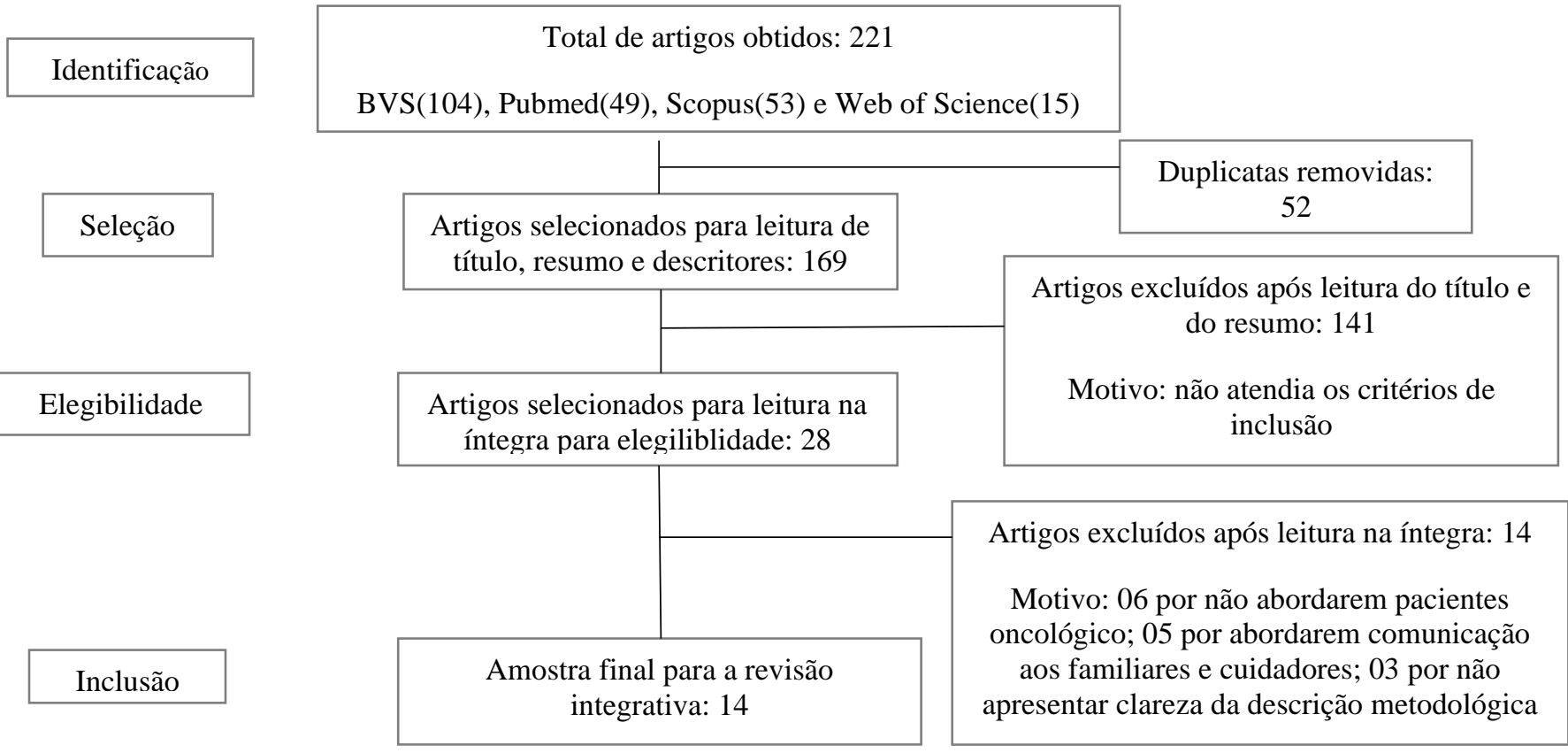

Fonte: Dados da pesquisa, Rio de Janeiro/RJ, Brasil (2020).

Os artigos selecionados foram lidos e categorizados por dois revisores, a partir de um instrumento elaborado pelos mesmos, no qual foram incluídas as informações de cada estudo, com o objetivo de sintetizar e agrupar as informações, sendo extraídas dos artigos as seguintes variáveis: autor, ano de publicação, periódico, método e classificação do nível de evidências (Melnyk \& Fineout-Overholt, 2015), conforme o Quadro 2. 
Quadro 2 - Síntese das características dos estudos selecionados com o Autor, ano, periódico, método e classificação de nível de evidência.

\begin{tabular}{|c|c|c|c|c|}
\hline $\mathbf{N}$ & Autores/Ano & Periódico & Método & $\begin{array}{c}\text { Nível de } \\
\text { evidência }\end{array}$ \\
\hline 1 & Visser, M., Deliens, L., Houttekier,D./2014 & Critical Care & Revisão sistemática & I \\
\hline 2 & Munhoz, B. A., et al./2014 & Eisntein & Transversal & VI \\
\hline 3 & Anderson, W. G., Kools, S., Lyndon,A./2013 & Qualitative Health Research & Estudo qualitativo & VI \\
\hline 4 & Brom, L. et al./2014 & PloS one & Estudo qualitativo & VI \\
\hline 5 & $\begin{array}{l}\text { Enzinger, A. C., Zhang, B., Schrag, D., } \\
\text { Prigerson, H. G. /2015 }\end{array}$ & Journal of clinical oncology & Coorte & IV \\
\hline 6 & Pfeil, T. A., et al./2015 & The Oncologist & Estudo qualitativo & VI \\
\hline 7 & Salins, N., et al./2016 & Indian journal of palliative care & Transversal & VI \\
\hline 8 & Gramling, R., et al./ 2016 & JAMA Onco & Transversal & VI \\
\hline 9 & Oostendorp, L. J., et al./2016 & Journal of Cancer Education & $\begin{array}{l}\text { Estudo Clínico, } \\
\text { Randomizado }\end{array}$ & II \\
\hline 10 & Epstein, A. S., et al./2016 & Journal of Clinical Oncology & Coorte & IV \\
\hline 11 & $\begin{array}{l}\text { Galvão, M. I. Z., Borges, M.D.S., Pinho, D. } \\
\text { L. M./2017 }\end{array}$ & Revista Baiana de Enfermagem & Estudo qualitativo & VI \\
\hline 12 & Rodenbach, R. A., et al./2017 & Journal of Clinical Oncology & $\begin{array}{c}\text { Estudo } \\
\text { clínico randomizado }\end{array}$ & II \\
\hline 13 & Kawaguchi, S., et al./ 2017 & $\begin{array}{c}\text { American Journal of Hospice and } \\
\text { Palliative Medicine }\end{array}$ & Estudo qualitativo & VI \\
\hline 14 & Wittenberg, E., et al./2018 & Patient education and counseling & Estudo qualitativo & VI \\
\hline
\end{tabular}

Fonte: Dados da pesquisa, Rio de Janeiro/RJ, Brasil (2020).

Das 14 publicações incluídas, 1 (7\%) é artigo de revisão e 13 (93\%) são originais. Constatou-se que nos anos de 2014 e 2016 foram de maiores publicações sobre a temática, com 4 publicações cada, seguidos do ano de 2017 e 2015 com 3 e 2 artigos publicados respectivamente. Por último foi o ano de 2018 com 1 publicação,

Obteve-se $2(14 \%)$ publicações nacionais e $12(86 \%)$ foram publicações internacionais, o que confere pouca discussão sobre o tema na realidade brasileira. Quanto aos periódicos, 1 (7\%) foi publicado em periódico de enfermagem e os demais (93\%) em periódicos médicos. Dentre os periódicos encontrados, o que mais se destacou foi a Journal of Clinical Oncology com três $(21 \%)$ publicações.

Dos 14 artigos, 6 (43\%) abordam sobre barreiras enfrentadas por profissionais de saúde na comunicação da transição do paciente oncológico para os cuidados paliativos, $8(57 \%)$ exploram estratégias para uma comunicação de qualidade na transição para os cuidados paliativos.

Quanto ao nível de evidência observou-se que 64\% dos estudos eram do tipo VI, ou seja, evidências derivadas de um único estudo descritivo ou qualitativo; $14 \%$ eram do tipo II referente a evidências derivadas de pelo menos um ensaio clínico randomizado controlado bem delineado, $14 \%$ do nível IV relativo a evidências provenientes de estudos de coorte e de casocontrole bem delineados e 7\% classificados como nível I, derivado de evidências provenientes de revisão sistemática ou metanálise de todos os relevantes ensaios clínicos randomizados controlados ou oriundas de diretrizes clínicas baseadas em revisões sistemáticas de ensaios clínicos randomizados controlados. 
Os artigos selecionados foram categorizados em dois temas principais acerca da comunicação na transição para os cuidados paliativos oncológicos, conforme Minayo, Deslandes e Gomes (2016): 1- Barreiras da comunicação na transição para os cuidados paliativos 2- Estratégias para uma comunicação eficaz na transição para os cuidados paliativos. Segue abaixo os Quadros 3 e 4 com resumo dos resultados acerca da comunicação na transição para os cuidados paliativos de acordo com cada tema categorizado:

Quadro 3 - Artigos categorizados ao tema 1: Barreiras da comunicação na transição para os cuidados paliativos-Rio de Janeiro, RJ, Brasil, 2020.

\begin{tabular}{|c|c|}
\hline Autores/Ano & Desfecho dos Resultados \\
\hline $\begin{array}{l}\text { Anderson, W. G., Kools, S., } \\
\text { Lyndon, A./2013 }\end{array}$ & $\begin{array}{l}\text { Embora médicos e pacientes reconhecessem a gravidade do câncer, raramente reconheciam a } \\
\text { possibilidade da morte, o que dificultava discussões sobre final de vida, objetivos, medos, esperanças e } \\
\text { opções de tratamento. }\end{array}$ \\
\hline Gramling, R., et al./ 2016 & $\begin{array}{l}\text { Atestou a discordância de informações entre paciente e médico quanto ao prognóstico do câncer, } \\
\text { identificando assim um marcador de comunicação inadequada, o que dificultou a tomada de decisão do } \\
\text { paciente sobre o tratamento. }\end{array}$ \\
\hline Oostendorp, L. J., et al./2016 & $\begin{array}{l}\text { Validou que muitos pacientes com câncer avançado gostariam de saber informações detalhadas sobre } \\
\text { riscos e benefícios relacionados ao tratamento paliativo, porém a maioria dos oncologistas tiveram } \\
\text { dificuldades com pacientes que desejavam informações acerca da sobrevivência. }\end{array}$ \\
\hline $\begin{array}{l}\text { Enzinger, A. C., Zhang, B., } \\
\text { Schrag, D., Prigerson, H. G. } \\
/ 2015\end{array}$ & $\begin{array}{l}\text { Os pacientes oncológicos são mais otimistas quanto a expectativa de vida. Uma compreensão realista é } \\
\text { necessária para corrigir os equívocos, sem prejudicar o bem estar emocional, isso pode facilitar o } \\
\text { planejamento do cuidado. }\end{array}$ \\
\hline Pfeil, T. A., et al./2015 & $\begin{array}{l}\text { Três barreiras da comunicação foram identificados: incerteza sobre o momento certo, incerteza sobre } \\
\text { quem é responsável por iniciar discussões e incerteza sobre o equilíbrio entre evidências objetivas, } \\
\text { desejos do paciente e seu julgamento subjetivo. }\end{array}$ \\
\hline $\begin{array}{l}\text { Visser, M. Deliens, L., } \\
\text { Houttekier,D./2014 }\end{array}$ & $\begin{array}{l}\text { Relata diversas barreiras comunicacionais relacionadas ao médico que dificulta a comunicação adequada } \\
\text { e tomada de decisão em UTIs. }\end{array}$ \\
\hline
\end{tabular}

Fonte: Autores (2020). 
Quadro 4 - Artigos categorizados ao tema 2: Estratégias para uma comunicação eficaz na transição para os cuidados paliativos-Rio de Janeiro, RJ, Brasil, 2020.

\begin{tabular}{|c|c|}
\hline Autores/Ano & Desfecho dos resultados \\
\hline Munhoz, B. A., et al./2014 & $\begin{array}{l}\text { A qualidade e frequência da comunicação mediada pela mútua confiança entre profissional-paciente-família } \\
\text { foram fundamentais para satisfação dos cuidadores e pacientes oncológicos durante toda a trajetória da } \\
\text { doença. }\end{array}$ \\
\hline Epstein, A. S., et al./2016 & $\begin{array}{l}\text { Discussões prognósticas mais recentes são importantes para melhorar a compreensão dos pacientes sobre o } \\
\text { estágio da doença. }\end{array}$ \\
\hline Rodenbach, R. A., et al./2017 & $\begin{array}{l}\text { Uma sessão prévia de "coaching" em comunicação ajudou os pacientes e seus cuidadores a procurar } \\
\text { ativamente informações e questionar mais sobre seu prognóstico. }\end{array}$ \\
\hline Salins, N., et al./2016 & $\begin{array}{l}\text { Oncologista, enfermeiros e pacientes reconheceram os benefícios da integração com os cuidados paliativos } \\
\text { para a melhora no controle de sintomas, comunicação prognóstica e discussões sobre a limitação do } \\
\text { tratamento. }\end{array}$ \\
\hline Brom, L., et al./2014 & $\begin{array}{l}\text { Comunicação sobre as expectativas e preferências dos pacientes quanto futuras decisões de tratamento pode } \\
\text { auxilia-los e melhorar a tomada de decisão. }\end{array}$ \\
\hline $\begin{array}{l}\text { Galvão, M. I. Z., Borges, } \\
\text { M.D.S., Pinho, D. L. } \\
\text { M./2017 }\end{array}$ & $\begin{array}{l}\text { Reafirmou a importância de técnicas e estratégias que facilitem a participação ativa do paciente no } \\
\text { enfretamento do câncer. }\end{array}$ \\
\hline Kawaguchi, S., et al./2017 & Apontou necessidades teóricas e práticas para melhorar habilidades comunicacionais em cuidados paliativos. \\
\hline Wittenberg, E., et al./2018 & $\begin{array}{l}\text { O curso de comunicação COMFORT forneceu habilidades de comunicação essenciais para enfermeiros } \\
\text { oncologistas. Tais habilidades se mostraram importantes pra um atendimento de qualidade e centrada no } \\
\text { paciente em todo o tratamento do câncer. }\end{array}$ \\
\hline
\end{tabular}

Fonte: Autores (2020).

\section{Discussão}

\section{Barreiras da comunicação na transição para os cuidados paliativos}

Embora os cuidados paliativos sejam muito valiosos em toda a trajetória da doença, as questões sobre fim de vida é uma das discussões mais desafiadoras, que inclui falar sobre prognóstico, preferências e prioridades em relação a tratamentos prolongados, questões de vida, incluindo metas e valores, bem como esperanças e medos em relação à morte(Visser, Deliens \& Houttekier, 2014; Anderson,Kools \& Lyndon, 2013; Gramling et al., 2016;Oostendorp et al., 2016).

Comunicar o paciente sobre o agravamento da doença e consequente transição para os cuidados paliativos, é considerado um momento complexo por muitos profissionais, já que esse pode simbolizar um sinal de desinvestimento no paciente, além de desencadear em desafios éticos, assim como lidar com sentimentos de desesperança e desapontamentos do paciente (Enzinger et al., 2015; Pfeil et al., 2015; Oostendorp et al., 2016). Todavia é uma ferramenta fundamental para redesenhar objetivos e metas não só de tratamento, mas de vida, que acontece em meio às incertezas, confusões e muitas vezes terror, considerado uma conversa não somente de metas de tratamento, mas principalmente de valores pessoais, a qual ajuda o paciente priorizar seu tempo limitado para suas reais necessidades (Enzinger et al., 2015; Gramling et al., 2016).

Apesar disso, a comunicação e decisões sobre cuidados no final da vida são frequentemente realizados tardiamente, onde médicos, bem como enfermeiros se sentem angustiados pela percepção de cuidados inadequados (Visser, Deliens \& 
Houttekier, 2014). Além disso, pode ser visto através das evidências que pacientes e familiares esperam muitas vezes o médico iniciar a conversa sobre os cuidados paliativos por sua vez, médicos também aguardam os pacientes e familiares solicitarem tal conversa. Essa espera de ambas as partes pode virar um ciclo perpétuo de não discussão, o qual é alimentado por múltiplas barreiras relacionadas aos profissionais como medo do impacto emocional sobre o paciente, destruindo a esperança, sentir-se despreparado para informar honestamente, falta de treinamento em comunicação e falta de consenso entre a equipe sobre prognóstico, tratamento e tomada de decisão no fim da vida (Visser, Deliens \& Houttekier, 2014; Anderson, Kools \& Lyndon, 2013; Enzinger et al., 2015; Pfeil et al., 2015).

Com isso, observou-se que a comunicação na transição para os cuidados paliativos muitas vezes não ocorre ou é falha, o que resulta na incompreensão acerca do prognóstico e consequentemente do próprio cuidado paliativo. É um momento caracterizado muitas vezes por discrepâncias entre o conhecimento do paciente e do médico sobre a doença, configurando assim, um potencial marcador de comunicação inadequada (Anderson, Kools \& Lyndon, 2013; Enzinger et al., 2015; Pfeil et al., 2015; Gramling et al., 2016; Oostendorp et al., 2016). Ao mesmo tempo, tem sido identificado que os pacientes desejam receber informação sobre possibilidades de cura ou não, relacionando o acesso a esse tipo de informação a atendimento de alta qualidade (Visser, Deliens \& Houttekier, 2014; Anderson, Kools \& Lyndon, 2013; Enzinger et al., 2015; Gramling et al., 2016; Oostendorp et al., 2016).

Dessa maneira, múltiplas barreiras foram detectadas, as quais podem estar ligadas a fatores médicos e pacientes colaborando para uma comunicação inadequada.Todavia as barreiras mais encontradas ainda estão relacionadas à atitude dos profissionais, já que esses compreendem que seu trabalho é salvar vidas ao invés de fornecer uma melhor qualidade de vida, até onde for possível, o que promove tratamentos de sustentação de vida muitas vezes prolongados e desnecessários, baseados apenas na decisão médica, favorecendo a falta de visão holística e impedindo a compreensão do paciente sobre quais são seus interesses nesse momento (Visser, Deliens \& Houttekier, 2014; Anderson,Kools \& Lyndon, 2013; Pfeil et al., 2015; Oostendorp et al., 2016).

\section{Estratégias para uma comunicação eficaz na transição para os cuidados paliativos}

A comunicação entre a equipe-paciente-família é essencial para promover um cuidado holístico, a qual envolve compartilhar informações, pensamentos e sentimentos. Essa é considerada eficaz quando a interação transmite a mensagem pretendida, a qual dependendo da forma que é transmitida pode motivar a aceitação ou resistência ao que está sendo informado, influenciando diretamente na aceitação e atitudes diante do diagnóstico, prognóstico e tratamento proposto (Galvão, Borges\&Pinho,2017). Por isso é importante intervir na melhoria da qualidade de comunicação com foco centrado no paciente e família, visto que isso ajudaria a satisfazer as necessidades dos pacientes e auxiliaria em sua futura tomada de decisão (Brom et al., 2014).

Como estratégia primária para uma comunicação eficaz entre profissionais e pacientes, é preciso que a educação em cuidados paliativos seja melhor ensinada nas academias, explorando teorias e sessões práticas para aprimoramento das habilidades comunicacionais na formação dos profissionais, além de realizar mais estudos que possibilitem alinhavar melhor a postura que profissionais de saúde devem ou não assumir ao comunicarem ao paciente e família quanto a transição para os cuidados paliativos (Munhoz et al., 2014; Kawaguchi et al., 2017).

Para que haja uma comunicação efetiva é necessário atenção, linguagem adequada e doação de tempo, sendo primordial para o paciente ter confiança em seu médico e toda equipe de saúde que faz parte desse cuidado, é preciso ser acolhido de forma digna e humanizada, onde obter informações sobre sua doença é uma questão prioritária nesse processo, desde o diagnóstico até as fases mais avançadas da doença (Munhoz et al., 2014; Galvão, Borges \& Pinho,2017). 
Dessa maneira, uma das estratégias a ser priorizada para melhor qualidade na comunicação é que o anúncio da má notícia ocorra sempre em um local reservado, o qual viabilize a individualidade e privacidade para expressão de pensamentos e que essa sempre tenha a presença da tríade equipe-família-paciente (Galvão, Borges \& Pinho,2017).

$\mathrm{O}$ trabalho em equipe formada por diferentes profissionais e recursos melhora a efetividade e resolutividade dos serviços de saúde, principalmente quando há integração dos cuidados ativos com os cuidados paliativos (Salins et al., 2016).

Nesse contexto, oportunizar discussões sobre a realidade da doença avançada é de importância ímpar para individualização do tratamento. Essa comunicação se torna fundamental para tomar decisões futuras quanto a terapêutica, pois ajuda a conhecer as prioridades desses garantindo sua tomada de decisão além de facilitar o planejamento antecipado de cuidados e o ajuste dos objetivos. É uma estratégia que requer equilíbrio entre fornecer informações suficientes, sem fornecer informações indesejadas pelo paciente, contudo, evita tratamentos agressivos e onerosos, além de internações desnecessárias, contribuindo para melhor qualidade de vida (Visser, Deliens \& Houttekier, 2014; Oostendorp et al., 2016, Rodenbach et al., 2017; Wittenberg et al., 2018).

Para garantir a individualização do tratamento e colaborar para uma comunicação mais clara entre profissional de saúde e paciente é preciso saber as expectativas e reais necessidades desse, já que a comunicação sobre desejos e preferências dos pacientes é de grande importância para conhecer as prioridades do paciente e garantir sua tomada de decisão quanto ao tratamento (Munhoz et al., 2014; Pfeil et al., 2015). Para isso é preciso que a equipe estimule o paciente a reconhecer seus sentimentos e a elaborar estratégias para adaptar-se a situação através da ressignificação da doença e assim alcance sua autonomia dentro das suas limitações (Galvão, Borges \& Pinho,2017; Wittenberg et al., 2018).

Apesar da fase de transição para os cuidados paliativos ser uma fase estratégica para os profissionais compreenderem os desejos e necessidades de informação referidos pelos pacientes, é importante relatar que o ideal é que o paciente receba os cuidados paliativos junto com o tratamento ativo dirigido à doença, pois isso possibilita melhor controle de sintomas, melhores comunicações sobre a saúde e capacita pacientes e familiares na tomada de decisões (Salins et al., 2016; Kawaguchi et al., 2017).

Dessa maneira, as estratégias para uma melhor qualidade na comunicação com pacientes com câncer avançado são primeiramente manter uma conversa aberta e honesta, focar nas metas do tratamento e no objetivo dos cuidados paliativos, tirando o foco da cura e proporcionando melhor qualidade de vida e controle de sintomas. É um instrumento de cuidado iniciar a comunicação o mais cedo possível e manter frequente, sendo realizada em diferentes momentos, já que às vezes não é possível para os pacientes e familiares absorverem e compreender toda a informação em um só encontro (Epstein et al., 2016; Galvão, Borges \& Pinho,2017).

\section{Conclusão}

Diante do exposto, conclui-se que a produção científica sobre comunicação na transição para os cuidados paliativos oncológicos está centrada em pesquisas com desenho descritivo/qualitativo, considerado baixo nível de evidência, onde verificou-se que há uma produção incipiente quanto a temática relacionada à realidade brasileira, sobretudo necessitando elaboração de estudos com melhor nível de evidência.

Os resultados apontaram que a complexidade da comunicação de notícias difíceis em cuidados paliativos requer atuação de toda equipe de saúde, um trabalho interdisciplinar, visto que o paciente com câncer avançado sofre de problemas físicos, emocionais, sociais e até mesmo espirituais, exigindo de todos os profissionais uma assistência holística as necessidades do cliente e da família diante das incertezas e dúvidas que marcam o momento da transição para os cuidados paliativos.

As categorias temáticas abordadas evidenciaram que a comunicação na transição para os cuidados paliativos ainda é um momento difícil e complexo para os profissionais de saúde, favorecendo barreiras comunicacionais entre a tríade equipe- 
cliente-família. Todavia é um momento estratégico para planejar e redesenhar objetivos de tratamento mesmo que já não haja possibilidade de cura, onde estratégias como uma comunicação honesta, o mais breve possível e contínua são importantes, porém, sobretudo deve-se respeitar as preferências do cliente para que haja uma comunicação eficaz.

Conforme os resultados obtidos, recomenda-se que sejam realizadas pesquisas que explorem a realidade brasileira sobre a comunicação na transição para os cuidados paliativos devido a lacuna encontrada de estudos nacionais sobre a temática em questão.

Vale ressaltar que, a presente revisão apresenta limitações, tais como o limite de cinco anos para inclusão dos estudos e inclusão apenas de artigos disponíveis online gratuitamente, os quais podem ter levado a dispensar a inclusão de alguns estudos, que poderiam ter sido investigados.

\section{Referências}

Abreu, W. C. (2017). De uma síndrome a condição social: cuidados paliativos a pessoas com demência avançada. Rev Rene, 18(1),1-2. https://doi.org/10.15253/2175-6783.2017000100001

Afonso, S. B. C., \& Minayo, M. C. S. (2017). Relações entre oncohematopediatras, mães e crianças na comunicação de notícias difíceis. Ciência Saúde Coletiva, 22(1),53-62.https://doi.org/10.1590/1413-81232017221.14592016

Almeida K. L. S., \& Garcia D. M. (2015). Use of communication strategies in palliative care in Brazil: integrative review. Cogitare enferm, 20 (4), 720-727. http://dx.doi.org/10.5380/ce.v20i4.3950

Anderson, W. G., Kools, S., \& Lyndon, A. (2013). Dancing around death: hospitalist-patient communication about serious illness. Qualitative Health Research, 23(1), 3-13.https://doi.org/10.1177/1049732312461728

Andrade, C. G., Costa, S. F. G., \& Lopes, M. E. L. (2013). Cuidados paliativos: a comunicação como estratégia de cuidado para o paciente em fase terminal. Ciência \& Saúde Coletiva, 18(9), 2523-2530. https://doi.org/10.1590/S1413-81232013000900006

Brom, L., Pasman, H. R., Widdershoven, G. A., Van der Vorst, M. J., Reijneveld, J. C., Postma, T. J., \& Onwuteaka-Philipsen, B. D. (2014). Patients' preferences for participation in treatment decision-making at the end of life: qualitative interviews with advanced cancer patients. PloS one, 9(6), e100435.http://dx.doi.org/10.1371/journal.pone.0100435

Butler, A., Hall, H., \& Copnell, B. (2016). A Guide to Writing a Qualitative Systematic Review Protocol to Enhance Evidence-Based Practice in Nursing and Health Care. Worldviews on Evidence-Based Nursing, 13(3), 241-249. https://doi.org/10.1111/wvn.12134

Desantis, C. E., Lin, C. C., Mariotto, A. B., Siegel, R. L., Stein, K. D., Kramer, J. L., \& Jemal, A. (2014). Cancer treatment and survivorship statistics. A Cancer Journal for Clinicians, 64(4), 252-271. https://doi.org/10.3322/caac.21235

Enzinger, A. C., Zhang, B., Schrag, D., \& Prigerson, H. G. (2015). Outcomes of Prognostic Disclosure: Associations With Prognostic Understanding, Distress, and Relationship With Physician Among Patients With Advanced Cancer. Journal of clinical oncology, 33(32), 3809-3816. http://dx.doi.org/10.1200/JCO.2015.61.9239

Epstein, A. S., Prigerson, H. G., O'Reilly, E. M., \& Maciejewski, P.K. (2016). Discussions of life expectancy and changes in illness understanding in patients with advanced cancer. Journal of Clinical Oncology, 34(20):2398-2403. http://dx.doi.org/10.1200/JCO.2015.63.6696

Galvão, M. I. Z., Borges, M. D. S., \& Pinho, D. L. M. (2017). Comunicação interpessoal com pacientes oncológicos em cuidados paliativos. Revista Baiana de Enfermagem, 31(3), e22290. http://dx.doi.org/10.18471/rbe.v31i3.22290

Gramling, R., Fiscella, K., Xing, G., Hoerger, M., Duberstein, P., Plumb, S., \& Epstein, R. M. (2016). Determinants of Patient-Oncologist Prognostic Discordance in Advanced Cancer. JAMA Onco, 2(11), 1421-1426. http://dx.doi.org/10.1001/jamaoncol.2016.1861

Instituto Nacional de Câncer José Alencar Gomes da Silva. (2017). Estimativa 2018: incidência de câncer no Brasil. INCA

Kawaguchi, S., Mirza, R., Nissim, R, \& Ridley, J. (2017). Internal medicine residents' beliefs, attitudes, and experiences relating to palliative care: a qualitative study. American Journal of Hospice and Palliative Medicine, 34(4):366-372. http://ascopubs.org/doi/pdf/10.1200/JCO.2016.68.5651

Kumata, C. S., Aparecida Borges, A., \& Dupas, G. (2015). Communicating bad news to family of hospitalized child. Ciencia, Cuidado e Saude, 14(4), 14111418.http://dx.doi.org/10.4025/cienccuidsaude.v14i4.25894

Lima, M. P. O., \& Oliveira, M. C. X (2015). Significados do cuidado de enfermagem para familiares de pacientes em tratamento paliativo. Rev Rene, 16(4), 593-602. https://doi.org/10.15253/2175-6783.2015000400017

Melnyk, B. M. \& Fineout-Overholt, E. (2015). Evidence-based practice in nursing \& healthcare: a guide to best practice. (3a ed.), Lippincot.

Minayo, M. C. S., Deslandes, S. F., \& GOMES, R. (2016). Pesquisa social: teoria, método e criatividade: Vozes.

Munhoz, B. A., Paiva, H. S., Abdalla, B. M. Z., Zaremba, G., Rodrigues, A. M. P., Carretti, M. R., \& Giglio, A. (2014). From one side to the other: what is essential? Perception of oncology patients and their caregivers in the beginning of oncology treatment and in palliative care. Einstein, 12(4), 485491.http://dx.doi.org/10.1590/S1679-45082014RC3091 
Research, Society and Development, v. 10, n. 4, e38210414302, 2021

(CC BY 4.0) | ISSN 2525-3409 | DOI: http://dx.doi.org/10.33448/rsd-v10i4.14302

Oostendorp, L. J., Ottevanger, P. B., Van de Wouw, A. J., Honkoop, A. H., Los, M., Van der Graaf, W. T., \& Stalmeier, P. F. (2016). Patients' preferences for information about the benefits and risks of second-line palliative chemotherapy and their oncologist's awareness of these preferences. Journal of Cancer Education, 31(3), 443-448. http://dx.doi.org/10.1007/s13187-015-0845-9

Pacheco, L. S. P., Santos, G. S., Machado, R., Granadeiro, D. S., Melo, N. G. S., \& Passos, J.P. (2020) The nurse's effective communication process with the patient in palliative care. Research, Society and Development, 9(8), e747986524. https://doi.org/10.33448/rsd-v9i8.6524

Pfeil, T. A., Laryionava, K., Reiter-Theil, S., Hiddemann, W., \& Winkler, E. C. (2015). What keeps oncologists from addressing palliative care early on with incurable cancer patients? an active stance seems key. The Oncologist, 20(1), 56. http://dx.doi.org/10.1634/theoncologist.2014-0031

Rodenbach, R .A., Brandes, K., Fiscella, K., Kravitz, R. L., Butow, P. N., Walczak, A., \& Epstein, R. M. (2017). Promoting end-of-life discussions in advanced cancer: effects of patient coaching and question prompt lists. Journal of Clinical Oncology, 35(8): 842.

Salins, N., Patra, L., Rani, M. R. U., Lohitashva, S. O., Rao, R., Ramanjulu, R., \& Vallath, N. (2016). Integration of early specialist palliative care in cancer care: survey of oncologists, oncology nurses, and patients. Indian journal of palliative care, 22(3), 258. http://dx.doi.org/10.4103/0973-1075.185030

Sawada, N. O., Paiva, B. S. R., Paiva, C.E., Sonobe, H. M., García-Caro, M. P., \& Cruz-Quintana, F. (2017). Transcultural approach to the health-related quality of life of cancer patients. Rev Rene, 18(3), 368. https://doi.org/10.15253/2175-6783.2017000300012

Souza, M. T., Silva, M. D., \& Carvalho, R. (2010) Revisão integrativa: o que é e como fazer? Einstein, 8(1), 102-106. http://www.scielo.br/pdf/eins/v8n1/pt_1679-4508-eins-8-1-0102.pdf

Visser, M., Deliens, L., \& Houttekier, D. (2014). Physician-related barriers to communication and patient- and family-centred decision-making towards the end of life in intensive care: a systematic review. Critical Care, 18(6), 1-19 .http://dx.doi.org/10.1186/s13054-014-0604-z

Wittenberg, E., Ferrell, B., Goldsmith, J., Ragan, S. L., \& Buller, H. (2018). COMFORT ${ }^{\mathrm{TM}}$ SM communication for oncology nurses: Program overview and preliminary evaluation of a nationwide train-the-trainer course. Patient education and counseling, 101(3), 467-474. https://doi.org/10.1016/j.pec.2017.09.012 